\title{
Vorläufige Tagungsplanung des AEI für das Jahr 2009
}

Thema: Europäisierung messen jenseits des 80\%-Mythos

Datum: Frühjahr 2009

Ort: /

Betreuung: Dr. Dieter Plehwe, Dr. Annette Elisabeth Töller

Thema: Modulierte Mitgliedschaften

Datum: April 2009

Ort: Krakau

Betreuung: Prof. Dr. Dr. h.c. Kazimierz Lankosz

Thema: Europäische Verfassungsentwicklung

Datum: Juni 2009

Ort: $\quad$ Budapest

Betreuung: Prof. Dr. Oliver Diggelmann

Thema: Grundlagen einer europäischen Privatrechtsgemeinschaft

Datum: Juni 2009

Ort: $\quad$ Nancy

Betreuung: Prof. Dr. Christian Baldus, Prof. Dr. Dr. h.c. mult. Peter-Christian Müller-Graff

Thema: Anti-Korruptionsregime in Europa

Datum: 24. Juli 2009

Ort: Konstanz

Betreuung: Dr. Sebastian Wolf

Thema: Charitable giving in Europe

Datum: Oktober 2009

Ort: $\quad$ Mannheim

Betreuung: Dr. Friedrich Heinemann

Thema: Nationale Parlamente in der Europäischen Union

Datum: Oktober 2009

Ort: $\quad$ Berlin

Betreuung: Dr. Andreas Maurer, Matthias Heddenhausen

Thema: Kleine Mitgliedstaaten in der Europäischen Union (10. Drei-LänderTagung)

Datum: Oktober 2009

Ort: Wien

Betreuung: Prof. Dr. Stefan Griller, Prof. Dr. Fritz Breuss

Thema: Jahreskolloquium 2009

Datum: November 2009

Ort: /

Betreuung: / 
Thema: Die Arktispolitik der Europäischen Union

Datum: Dezember 2009

Ort: $\quad$ Berlin

Betreuung: Dr. Andreas Maurer

Thema: The Euro and the Structural Conflict in a Monetary Union - A Blueprint for Asia?

Datum: /

Ort:

Betreuung: Prof. Dr. Ansgar Belke, Prof. Dr. Mariusz K. Krawczyk

Thema: Polen im europäischen Integrationsprozess

Datum: /

Ort:

Betreuung: Prof. Dr. Beate Neuss, Dr. Burkard Steppacher

Adresse der Geschäftsstelle: AEI, c/o SWP, Ludwigkirchplatz 3-4, 10719 Berlin; Tel.: (030) 88920 186; Fax: (030) 88920 196; E-Mail: aei@aei-ecsa.de; im Internet: http://www.aeiecsa.de

\section{Studies on the European Union}

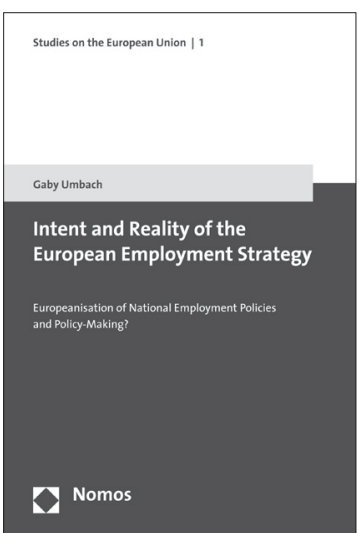

Intent and Reality of the European

Employment Strategy

Europeanisation of National Employment Policies and Policy-Making?

Von Dr. Gaby Umbach

2008, Band 1, 487 S., brosch., 59,- $€$,

ISBN 978-3-8329-4128-4

Das vorliegende Buch beschäftigt sich intensiv mit den unterschiedlichen Facetten der EBS und ihrer Wirkung. Es geht dabei über bisherige Einzelstudien hinaus und befasst sich nicht nur mit deren Entstehung, Entwicklung und Merkmalen. Das Werk kontrastiert vielmehr den eigenen Anspruch der EBS mit ihrer politischen Realität und untersucht theoretisch hoch reflektiert deren Einfluss. 